\title{
Flora da Bahia: Menyanthaceae
}

\author{
Ana Maria Giulietti ${ }^{1,2,3^{*}}$, Tasciano dos Santos Santa Izabel $^{1, \mathrm{~b}}$ \& Reyjane Patrícia de Oliveira $^{1, \mathrm{c}}$ \\ ${ }^{1}$ Programa de Pós-graduação em Botânica, Departamento de Ciências Biológicas, Universidade Estadual de Feira \\ de Santana, Feira de Santana, Bahia, Brasil. \\ ${ }^{2}$ Herbarium, Royal Botanic Gardens, Kew, Reino Unido. \\ ${ }^{3}$ Instituto Tecnológico Vale, Belém, Pará, Brasil.
}

\begin{abstract}
Resumo - É apresentado o levantamento florístico de Menyanthaceae da Bahia, Brasil, como contribuição ao conhecimento da flora do Estado. Apenas Nymphoides humboldtiana foi reconhecida para a Bahia; N. indica, previamente citada para o Brasil, está restrita ao Velho Mundo. São apresentadas descrições da família, do gênero e da espécie, além de ilustrações e comentários gerais sobre a espécie.

Palavras-chave adicionais: Brasil, florística, Nymphoides, taxonomia.
\end{abstract}

\begin{abstract}
Flora of Bahia: Menyanthaceae) - The floristic survey of the Menyanthaceae from Bahia, Brazil, is presented as a further contribution to the knowledge of the Flora of that State. Only Nymphoides humboldtiana was recognized for Bahia; $N$. indica, previously cited for Brazil, is restricted to the Old World. Descriptions of the family, genus and species, illustrations, and general notes on the species are presented.
\end{abstract}

Additional key words: Brazil, floristics, Nymphoides, taxonomy.

\section{MeNYANTHACEAE}

Ervas aquáticas ou semiaquáticas, anuais ou perenes, rizomatosas. Folhas alternas, simples, reniformes, cordiformes, orbiculares, largamente ovais, lineares ou compostas 3-folioladas, geralmente peltadas, sem estípulas. Inflorescência racemosa, fasciculada, ou raramente flores solitárias. Flores bissexuadas, geralmente distílicas, ou unissexuadas, diclamídeas, pentâmeras, actinomorfas; cálice com sépalas livres ou unidas na base; corola gamopétala, prefloração valvar ou induplicado-valvar, lobos com margem fimbriada ou cristada; androceu isostêmone, estames alternos às pétalas, epipétalos, anteras 2-tecas, sagitadas, rimosas, versáteis, grãos de pólen 3colporados; gineceu 2-carpelar, ovário súpero ou semiínfero, 1-locular, geralmente com disco nectarífero na base, óvulos anátropos, placentação parietal, estilete simples, estigma 1- ou 2-lobado. Fruto cápsula, raramente baga. Sementes geralmente numerosas, endosperma abundante, oleoso, embrião linear.

A família tem distribuição cosmopolita e inclui de 30 a 35 espécies, reunidas em cinco gêneros, sendo Nymphoides, aquele com maior número de espécies (Kadereit 2007; Tippery et al. 2008).

\section{Nymphoides Ség.}

Plantas aquáticas. Folhas flutuantes, orbiculares ou largamente ovais, peltadas, base cordada. Flores em fascículos axilares, distílicas, frágeis, perfumadas; cálice com sépalas unidas, persistente no fruto; corola

\footnotetext{
*Autora para correspondência: anagiulietti@hotmail.com; atazuefsbot@gmail.com; ${ }^{b}$ rpatricia@uefs.br;

Editor responsável: Abel Augusto Conceição

Submetido: 7 maio 2015; aceito: 21 out. 2015

Publicação eletrônica: 19 nov. 2015; versão final: 2 dez. 2015
}

com pétalas amarelas ou brancas, tubo curto, lobos lanceolados, pilosos internamente, estames com anteras ovais ou lineares; ovário súpero, com poucos a muitos óvulos, disco hipogíneo glandular, estilete curto, estigma 2-lobado a capitado. Sementes com testa lisa, foveolada, tuberculada ou ciliada.

Gênero pantropical com cerca de 20 espécies, de ocorrência nas Américas, África, Austrália e Ásia, tendo nesses dois últimos continentes sua maior diversidade. Apresenta taxonomia complexa devido à presença de híbridos e séries poliploides, sendo a testa da semente um importante caráter taxonômico (Sivarajan et al. 1984; Yatskievych 2001; Kadereit 2007; Tippery \& Les 2011). Apenas Nymphoides humboldtiana ocorre no estado da Bahia e no Brasil.

Nymphoides humboldtiana (Kunth) Kuntze, Revis. Gen. P1. 2: 429. 1891.

Figuras 1-3.

Ervas rizomatosas, com ramos estoloníferos. Folhas com pecíolo 9-27 cm compr., limbo flutuante, largo-oval a orbicular, (3-)6-15 × (3-)6,5-15,5 cm, ápice emarginado, base cordada, lobos basais (1-)2$5,5 \mathrm{~cm}$ compr., membranáceo a ligeiramente cartáceo, discolores, face adaxial verde, face abaxial vermelhoarroxeada, coberta por glândulas pequenas, geralmente portando galhas. Flores $8-25$, emergindo próximo à base do limbo, brevistilas e longistilas na mesma população, ca. 1,5 cm diâm.; pedicelo 1,7-5,5 cm compr.; sépalas 4-7 mm compr., verdes com ápice avermelhado; corola 1-1,5 cm compr., lobos 4 ou 5 , lanceolados, face adaxial branca com base amarelada, densamente pilosa, margem fimbriada; estames 4 ou 5 , filetes 3-8 $\mathrm{mm}$ compr., anteras enegrecidas; estilete linear, verde, 3-5 mm compr., estigma irregularmente capitado, amarelo-claro, ovário avermelhado, multiovulado. Cápsulas ovoides, ca. $6 \mathrm{~mm}$ compr. 


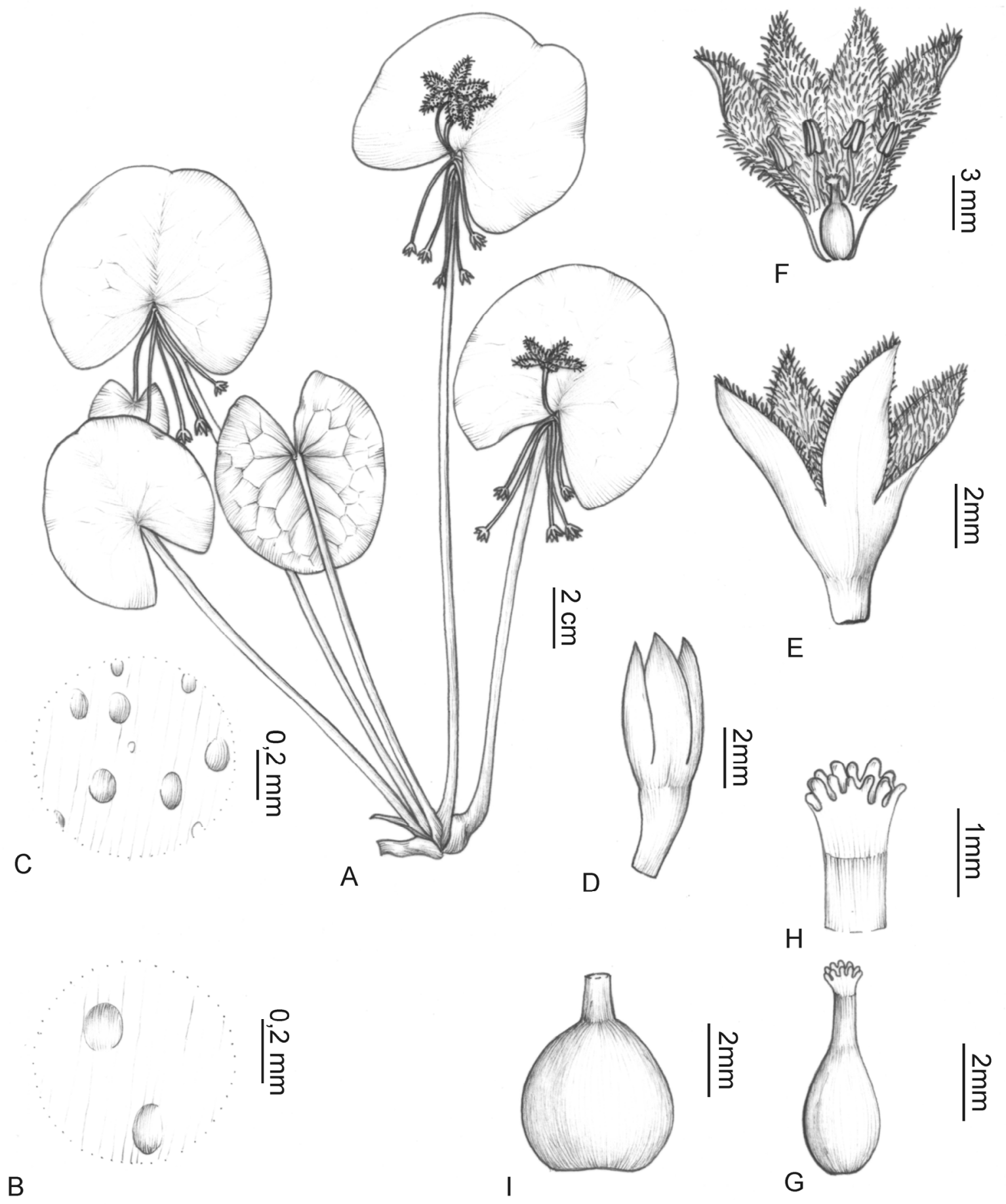

Figura 1. Nymphoides humboldtiana: A- hábito; B- detalhe da superfície adaxial da folha, mostrando galhas com ovos de insetos; C- detalhe da superfície abaxial da folha, mostrando galhas com ovos de insetos; D- botão floral; E- tubo e lobos da corola tetrâmera; F- flor tetrâmera e brevistila aberta, mostrando a face interna da corola, androceu e gineceu; G- gineceu brevistilo; H- detalhe do estigma irregularmente lobado; I- fruto jovem (Junqueira 16).

Distribuição neotropical, no Sul dos Estados Unidos, México, América Central e América do Sul, até o Uruguai (Progel 1865; Ordnuff 1969; Tippery \& Les 2011). Para o Brasil, Nymphoides humboldtiana vinha sendo identificada como $N$. indica (L.) Kuntze, com registros do Amazonas ao Rio Grande do Sul (Conceição \& Giulietti 1998; Souza \& Lorenzi 2008; Amaral 2015). D6, D7, D10, E6, E8, E9, E10, F6, F8, G7, G8 e H9: em lagoas permanentes ou temporárias associadas à Mata Atlântica, Caatinga e Cerrado. Floresce de outubro a fevereiro.

Material selecionado: Alagoinhas, $12^{\circ} 06^{\prime} 09^{\prime \prime} \mathrm{S}, 38^{\circ} 24^{\prime} 54^{\prime \prime} \mathrm{W}$, 22 ago. 2006, N.G. Jesus 869 (HUEFS); Alcobaça, 17³0'S, $39^{\circ} 13^{\prime} \mathrm{W}, 8$ dez. 1981, G.P. Lewis \& A.M. Carvalho 804 (K); Andaraí, 12 $2^{\circ} 48^{\prime} 26^{\prime} \mathrm{S}, 41^{\circ} 19^{\prime} 53^{\prime \prime}$ 'W, 19 nov. 2006, M.F. Costa 29

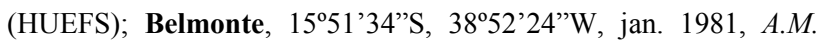

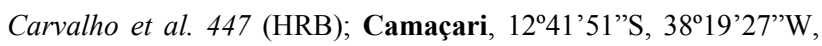
nov. 1995, A.C. Navano 26 (CEPEC); Candeias, $12^{\circ} 40^{\prime} 04^{\prime \prime}$, 
38 33'02”', 23 set. 2004, K.R.B. Leite et al. 444 (HUEFS); Caravelas, 17³0'35"S, 38¹1'28'W, 31 ago. 2011, E.N. Matos 522

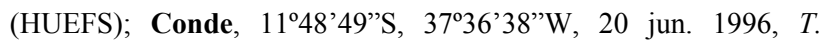
Ribeiro et al. 28 (HRB, HUEFS); Correntina, 1311'16"S, 4440'24”'W, 15 mar. 2010, K. Yoshida-Arns et al. 576 (HUEFS); Cruz das Almas, 12³9'11'S, 3907'19'W, 7 mar. 2008, L.Y.S, Aona et al. 1043 (HURB); Dias D'Ávila, 12³6'45”'S, 38¹7'49”'W,

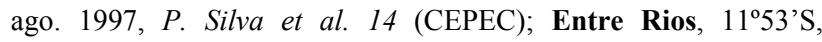
38²'W, 24 mar. 2013, A.V. Popovkin et al. 1393 (HUEFS); Feira de Santana, $12^{\circ} 16^{\prime} 00^{\prime \prime} \mathrm{S}$, 38 $58^{\circ} 00^{\prime \prime} \mathrm{W}, 29$ jan. 1999, M.E.R. Junqueira et al. 16 (HUEFS); Ilhéus, 1447'20”S, 3902'58'W, 6 Jun. 2002, E.A. Rocha et al. 967 (CEPEC, HUEFS); Itatim, 1242'49”'S, 3941'36”', 30 ago. 1997, E. Melo et al. 66 (HUEFS);

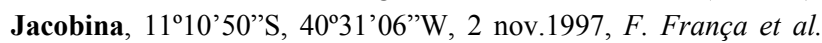
2458 (HUEFS); Lençóis, 1349'00”S, 4143'00”'W, 19 fev. 1999, M.P. Sena s.n. (HUEFS 36572); Morro do Chapéu, 11²4'22”'S,
41'22'17'W, 17 nov. 1999, E. Melo et al. 3150 (HUEFS); Mucugê, 1301'24”S, 41'25'45”W, 6 nov. 2007, E. Melo 5368 (HUEFS); Muritiba, 12'36'24"S, 39 00'45"W, maio 2000, F. França et al. 3333 (HUEFS); Piatã, 1249'16”S, 4148'42”W, 997 m s.n.m., 17 nov. 2007, J.L. Ferreira 226 (HUEFS); Pilão Arcado, 10¹0'2”S, 42 57'12”W, 20 mar. 2006, D. Carneiro-Torres et al. 637

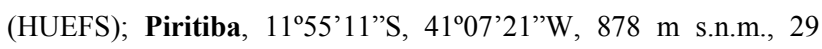
maio 2011, I.M. Souza 46 (HUEFS); Salinas de Margarida, 12055'16"S, 3846'26”'W, 29 set. 2011, E.N. Matos et al. 536 (HUEFS); Salvador, $12^{\circ} 56^{\prime}$ 'S, $38^{\circ} 21^{\prime} \mathrm{W}, 16$ jan. 1986, L.P. Queiroz 983 (HUEFS); Santo Antônio de Jesus, 1258'08'S, 39¹5'41'W, 2 ago. 2009, C.T. Lima 187 (HUEFS); São Felipe, 1247'02”S, 3905'29'W, 26 mar. 2013, L.Y.S. Aona et al. 2542 (HUEFS); São Sebastião do Passé, 12³0'45"S, 38²9'43”'W, mar. 1996, L.R. Noblick et al. 2575 (HRB). Valença, 13'22'26”'S, 3904'03”'W, out. 1998, G. Hatschbach et al. 68466 (ALCB); Vera Cruz,
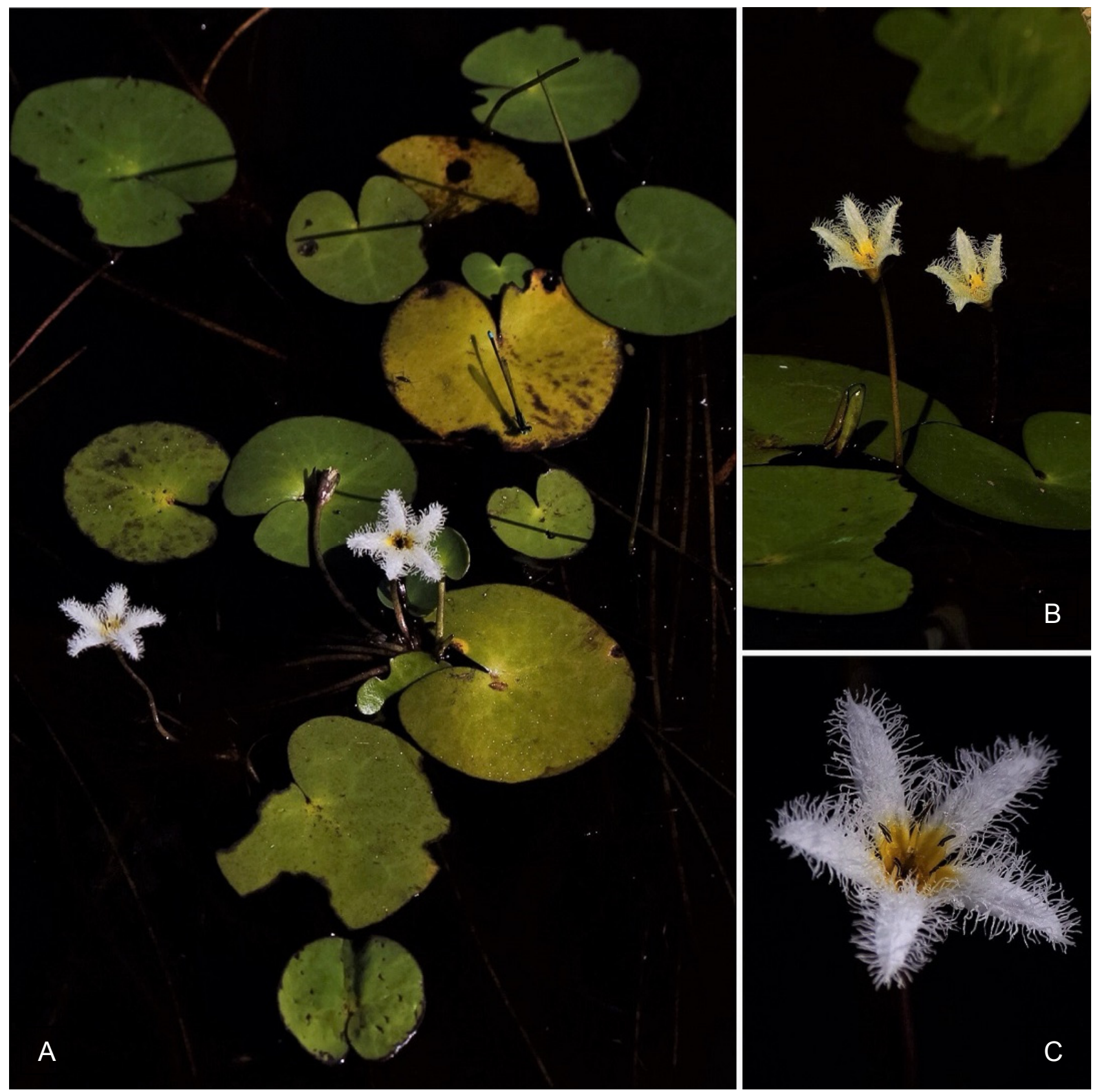

Figura 2. Nymphoides humboldtiana: A- hábito aquático; B- flores, vista lateral; C- flor, vista de cima (fotos: A. Rapini). 
1257’36”S, 38³6’32”W, abr. 1994, E. Melo \& F. França 943 (HUEFS); Vitória da Conquista, 14²51'58”S, 4050'22”'W, fev. 1972, T.S. Santos 2251 (CEPEC, K).

Na Flora Brasiliensis (Progel 1865), Nymphoides humboldtiana aparece na família Gentianaceae subf. Menyanthoideae, como Limnanthemum humboldtianum (Kunth) Griseb. Nas Floras de Santa Catarina (Fabris \& Klein 1971), da Serra do Cipó (Conceição \& Giulietti 1988), de São Paulo (Anderson \& Amaral 2005) e na Lista de Espécies da Flora do Brasil (Amaral 2015), foi considerada sinônimo de Nymphoides indica. Essa delimitação teve como base os trabalhos de Ornduff (1969, 1970), que verificou uma grande variação, tanto na morfologia floral como no crescimento e na floração da espécie, concluindo que não há diferença morfológica relevante entre as populações do Novo e do Velho Mundo, embora nas Américas elas sejam tetraploides e na África, Ásia e Austrália diploides. As filogenias apresentadas por Tippery \& Les (2011), por outro lado, mostraram que apesar de morfologicamente indistinguíveis, Nhymphoides indica e $N$. humboldtiana representam linhagens divergentes, refletindo um padrão geográfico em sua evolução. As amostras do Brasil, Equador e México formam um clado com as demais espécies americanas do gênero, enquanto as espécies das outras regiões emergem em outro clado. Assim, Tippery \& Les (2011) consideraram $N$. indica restrita ao Velho Mundo e $N$. humboldtiana restrita às Américas.

Na Bahia, N. humboldtiana apresenta ampla distribuição, ocorrendo desde o litoral, em áreas de restingas e de mata atlântica, até diversas áreas da Chapada Diamantina e nos cerrados do oeste do Estado. Apresenta grande variação morfológica, especialmente na dimensão e textura das folhas e na ocorrência de flores tetrâmeras e pentâmeras. Não há referência para a utilização da espécie no Estado, mas propriedades vermicidas, antissépticas, vermífugas, febrífugas e tônicas são referidas para a espécie (Hoehne 1948).

\section{AgRADECIMENTOS}

Aos curadores dos herbários ALCB, CEPEC, HRB, HUEFS e $\mathrm{K}$ pelo acesso às coleções e bibliotecas. À equipe do REFLORA em Kew, por todo o suporte logístico, especialmente à Catia Canteiro e Eimear Nic Lughadha. À FAPESB, pelo auxílio financeiro para o Projeto Flora da Bahia, processo APR0162/2007. Ao CNPq, pelo apoio financeiro aos projetos da Flora da Bahia (processos 562278/2010-9 e 483909/2012) e ao REFLORA (processo 563558/2010-5), incluindo auxílio na realização de visitas aos herbários e expedições de campo. AMG e RPO também agradecem ao CNPq pelas bolsas de Produtividade em Pesquisa (PQ Senior e PQ1D, respectivamente).

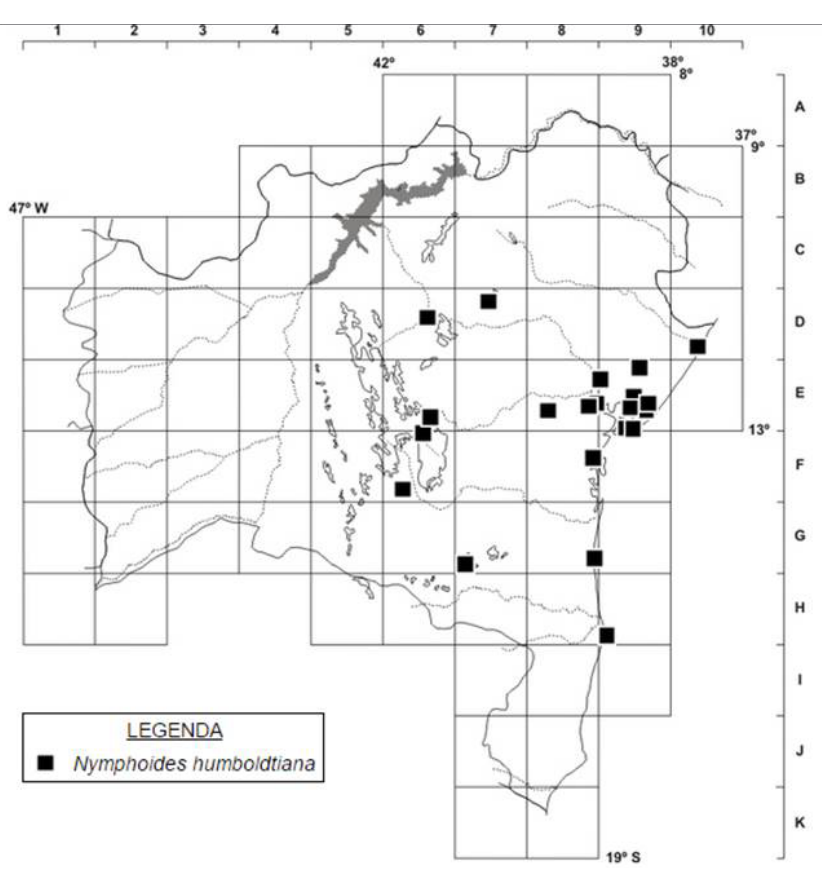

Figura 3. Mapa de distribuição de Nymphoides humboldtiana no estado da Bahia.

\section{REFERÊNCIAS}

Amaral, M.C.E. 2015. Menyanthaceae. In: Lista de Espécies da Flora do Brasil. Jardim Botânico do Rio de Janeiro. Disponível em <http://floradobrasil.jbrj.gov.br/jabot/floradobrasil/FB10053>; acesso em 7 maio 2015

Anderson, L.O. \& Amaral, M.C.E. 2005. Menyanthaceae. In: M.G.L. Wanderley, G.J. Shepherd, T.S. Melhem \& A.M. Giulietti (eds), Flora Fanerogâmica do Estado de São Paulo. Vol. 4. FAPESP/RiMa, São Paulo, p. 277-278.

Conceição, A.A. \& Giulietti, A.M. 1998. Flora da Serra do Cipó, Minas Gerais: Menyanthaceae. Boletim de Botânica da Universidade de São Paulo 17: 123-125.

Fabris, H.A \& Klein, R.M. 1971. Meniantáceas. In: R. Reitz (ed.), Flora Ilustrada Catarinensis. Herbário Barbosa Rodrigues, Itajaí.

Hoehne, F.C. 1948. Plantas Aquáticas. Instituto de Botânica, São Paulo.

Kadereit, J.W. 2007. Menyanthaceae. In: K. Kubitzki, J.W. Kadereit \& C. Jeffrey (eds), The Families and Genera of Vascular Plants. Vol. 8. Springer-Verlag, Berlin, p. 589-604.

Ornduff, R. 1969. Neotropical Nymphoides (Menyanthaceae): MesoAmerican and West Indian species. Brittonia 21: 346-351.

Ornduff, R. 1970. Citogeography of Nymphoides (Menyanthaceae). Taxon 19: 346-351.

Sivarajan, V.V.; Chaw, S. \& Joseph, K.T. 1989. Seed coat micromorphology of Indian species of Nymphoides (Menyanthaceae). Botanical Bulletin of Academia Sinica 30: 278-283.

Souza V.C. \& Lorenzi, H. 2008. Botânica Sistemática-guia ilustrado para identificação das famílias de fanerógamas nativas e exóticas no Brasil, baseado em APG II. Instituto Plantarum, Nova Odessa.

Tippery, N.P. \& Les, D.H. 2011. Phylogenetic relationships and morphological evolution in Nymphoides (Menyanthaceae). Systematic Botany 36(4): 11-1113. 
Tippery, N.P.; Les, D.H.; Padgett, D.J. \& Jacobs, W.L. 2008. Generic circumscription in Menyanthaceae: a phylogenetic evaluation. Systematic Botany 33: 598-612.

Tippery, N.P.; Les, D.H. \& Williams, C.R. 2011. Nymphoides humboldtiana (Menyanthaceae) in Uvalde County, Texas - a new record for the U.S.A. Journal of the Botany Research Institute of Texas 5(2): 889-890.

Yatskievych, K. 2001. Menyanthaceae. In: J.A. Steyermark, P.E. Berry \& B.H. Holst (eds), Flora of the Venezuelan Guayana. Vol. 6. Missouri Botanic Garden Press, St. Louis, p. 578-579.

\section{LISTA DE EXSICATAS}

Adorno, J.M.S. 3; Aona, L.Y.S. 1043, 2542; Carneiro-Torres, D. 637; Carvalho, A.M. 447; Conceição, A.A. 2309; Costa, M.F. 29; Ferreira, J.L. 117, 226; França, F. 2355, 2458, 3104, 3333, 5596; Giulietti, A.M. 2693; Gomes, D.J. 18; Guedes, M.L. 1029; Harley, R. 3408, 16105, 17438, 18004; Hatschbach, G. 68466; Hind, D.J.N. 38; Jesus, N.G. 869, 2271, 2274, 2286, 1840; Junqueira, M.E.R. 16; Leite, K.R.B. 444; Lewis, G.P. \& Carvalho, A.M. 804; Lima, C.T. 187; Matos, E.N. 522, 536; Mattos-Silva, L.A. 304; Melo, E. 66, 943 , 2117, 3150, 3767, 5368, 6456, 9974; Noblick, L.R. 2575, 2601; Navano, A.C. 26; Oliveira, I. 65,72; Pereira-Silva, G. 8458; Popovkin, A.V. 1393; Queiroz, L.P. 933; Ribeiro, T. 28; Rocha, E.A. 967; Santos, T.S. 2251; Sena, M.P. s.n. (HUEFS 36572); Silva, P. 14; Souza, I.M. 37, 46; Yoshida-Arns, K. 576. 